\title{
Comparison of Inflammation-Based Prognostic Scores in Patients undergoing Curative Resection for Non-small Cell Lung Cancer
}

\author{
Masaki Tomita $^{\mathrm{a}, \mathrm{c}}$, Takanori Ayabe ${ }^{\mathrm{a}}$, Ryo Maeda ${ }^{\mathrm{a}}$, Kunihide Nakamura ${ }^{\mathrm{b}}$
}

\begin{abstract}
Background: Inflammation-based prognostic scores, including Glasgow prognostic score (GPS), neutrophil-to-lymphocyte ratio (NLR), platelet-to-lymphocyte ratio (PLR), prognostic nutritional index (PNI), systemic immune-inflammation index (SII) and advance lung cancer inflammation index (ALI) are reported to be associated with survival in patients with non-small cell lung cancer (NSCLC). However, at present, there are no studies that compared these scoring systems for resectable NSCLC.
\end{abstract}

Methods: Three hundred forty-one NSCLC patients who underwent surgery at our institution were included. The optimal cut-off values of SII and ALI were calculated by the Cutoff Finder. The area under the receiver operating characteristics curve (AUC) was calculated to compare the predictive ability of each of the scoring systems. Univariate and multivariate analyses were performed to identify the clinicopathological variables associated with overall survival.

Results: The optimal cut-off value of SII and ALI were $471.2 \times 10^{9} / \mathrm{L}$ and 37.66, respectively. All scores were significantly related to the 5 -year cancer-specific survival. The ALI consistently had a higher AUC value in comparison with other inflammation-based prognostic scores. A multivariate analysis showed that GPS and ALI were independently associated with overall cancer-specific survival.

Conclusion: To the best of our knowledge, this is the first study to demonstrate that GPS and ALI appear to be superior to other inflammation-based prognostic scores in patients undergoing potentially curative resection for NSCLC.

Keywords: Inflammation-based prognostic scores; Non-small cell lung cancer; Surgery; Prognosis

Manuscript submitted March 16, 2018, accepted May 4, 2018

aDepartment of Thoracic and Breast Surgery, Faculty of Medicine, University of Miyazaki, Kihara 5200, Kiyotake, Miyazaki 889-1692, Japan

bDepartment of Cardiovascular Surgery, Faculty of Medicine, University of Miyazaki, Kihara 5200, Kiyotake, Miyazaki 889-1692, Japan

${ }^{\mathrm{c} C}$ Corresponding Author: Masaki Tomita, Department of Thoracic and Breast Surgery, Faculty of Medicine, University of Miyazaki, Kihara 5200, Kiyotake, Miyazaki 889-1692, Japan. Email: mtomita@med.miyazaki-u.ac.jp

doi: https://doi.org/10.14740/wjon1097w
Introduction

Recent studies have begun to unravel the mechanisms linking the host inflammatory response to tumor growth, invasion and metastasis in non-small cell lung cancer (NSCLC) [1].

It has been also recognized that systemic inflammatory responses play an important role in the development of tumor, enhancement of local immunosuppression, thereby worsening the prognosis of the patients [2-7]. Based on this relationship between inflammation and cancer progression, several inflammation-based scores, including Glasgow prognostic score (GPS) [2], neutrophil-to-lymphocyte ratio (NLR) [3], plateletto-lymphocyte ratio (PLR) [4], prognostic nutritional index (PNI) [5], systemic immune-inflammation index (SII) [6] and advance lung cancer inflammation index (ALI) [7], have been demonstrated to have prognostic value in NSCLC.

In other malignancies, some previous studies compared the prognostic value of selected inflammation-based scores [811]. To our knowledge, however, there are no studies elucidating which of these prognostic scores is more suitable in predicting outcomes in patients with NSCLC undergoing surgery. Therefore, the aim of this study was to compare the prognostic value of these inflammation-based scores in patients undergoing potentially curative resection for NSCLC.

\section{Patients and Methods}

This retrospective study examined the records of a sequential series of 381 patients with NSCLC who received complete resection and fill the inclusion criteria between January 2008 and December 2012 in our hospital. There were 173 men and 168 women (median age: 69 years old). The inclusion criteria were as following: histologically confirmed NSCLC and complete clinical, laboratory, imaging and follow-up data. The exclusion criteria included: preoperative chemotherapy/radiotherapy or died perioperative period, clinical evidence of infection or other bone marrow, hematological or autoimmune disease. This retrospective study was approved by Medical Ethics Committee of our hospital (O-0296). Complete blood count and routine biochemistry test, including serum C-reactive protein (CRP), serum albumin, serum carcinoembryonic antigen (CEA) and serum cytokeratin 19 fragments (CYFRA21-1), of each patient was applied 1 week before surgery. The GPS, NLR, PLR, PNI, 
Table 1. Inflammation-Based Prognostic Scores

\begin{tabular}{|c|c|}
\hline & Score \\
\hline \multicolumn{2}{|l|}{ Glasgow Prognostic Score (GPS) } \\
\hline C-reactive protein $\leq 10 \mathrm{mg} / \mathrm{L}$ and albumin $\geq 35 \mathrm{~g} / \mathrm{L}$ & 0 \\
\hline C-reactive protein $\leq 10 \mathrm{mg} / \mathrm{L}$ and albumin $<35 \mathrm{~g} / \mathrm{L}$ & 1 \\
\hline C-reactive protein $>10 \mathrm{mg} / \mathrm{L}$ and albumin $\geq 35 \mathrm{~g} / \mathrm{L}$ & 1 \\
\hline C-reactive protein $>10 \mathrm{mg} / \mathrm{L}$ and albumin $<35 \mathrm{~g} / \mathrm{L}$ & 2 \\
\hline \multicolumn{2}{|l|}{ Neutrophil lymphocyte ratio (NLR) } \\
\hline Neutrophil count/lymphocyte count $<5: 1$ & Low \\
\hline Neutrophil count/lymphocyte count $\geq 5: 1$ & High \\
\hline \multicolumn{2}{|l|}{ Platelet lymphocyte ratio (PLR) } \\
\hline Platelet count/lymphocyte count $<150: 1$ & 0 \\
\hline Platelet count/lymphocyte count 150 - 300 & 1 \\
\hline Platelet count/lymphocyte count $>300: 1$ & 2 \\
\hline \multicolumn{2}{|l|}{ Prognostic nutritional index (PNI) } \\
\hline Albumin $(\mathrm{g} / \mathrm{L})+5 \times$ total lymphocyte count $\geq 45$ & 0 \\
\hline Albumin $(\mathrm{g} / \mathrm{L})+5 \times$ total lymphocyte count $<45$ & 1 \\
\hline \multicolumn{2}{|l|}{ Systemic immune-inflammation index (SII) } \\
\hline Platelet count $\times$ NLR $<$ cut-off value & Low \\
\hline Platelet count $\times$ NLR $\geq$ cut-off value & High \\
\hline \multicolumn{2}{|l|}{ Advance lung cancer inflammation index (ALI) } \\
\hline Body mass index $\times$ albumin/NLR $\geq$ cut-off value & Low \\
\hline Body mass index $\times$ albumin/NLR $<$ cut-off value & High \\
\hline
\end{tabular}

SII and ALI were constructed as described in Table 1. Cut-off values of NLR, PLR and PNI were determined according to previous reports [3-5]. The optimal cut-off value of SII and ALI for overall cancer-specific survival was determined by Cutoff Finder (http://molpath.charite.de/cutoff) [12].

Association between categorical variables was assessed using the Fisher's exact test. Cancer-specific survival curves were plotted using the Kaplan-Meier method, with comparisons between groups performed using the log-rank test. A receiver operating characteristics (ROC) curve was generated and the area under the curve (AUC) was calculated to evaluate the discriminatory ability of each scoring systems. Univariate and multivariate analyses were performed for prognostic factors using the Cox proportional hazards model. Variables that proved to be significant on univariate analysis were subsequently subjected to a multivariate Cox proportional hazards model. Hazard ratios (HRs) estimated using the Cox analysis are reported as relative risks with corresponding $95 \%$ confidence intervals (CIs). Statistical analyses were performed using JMP (SAS Institute Inc., Cary, NC, USA). Differences were considered statistically significant when $\mathrm{P}<0.05$.

\section{Results}

Analysis using the Cutoff Finder showed the recommended cut-off values of SII and ALI for evaluating cancer-specific survival were $471.2 \times 10^{9} / \mathrm{L}$ and 37.66 , respectively.

The relationships between clinical characteristics and each inflammation-based score were shown in Table 2. There is a trend towards an association between these inflammationbased scores and gender, pStage and $\mathrm{pN}$ status. However, the results of these scores were not always identical each other.

The relationship between the inflammation-based prognostic scores and cancer-specific overall survival is shown in Figure 1. Based on total number of patients, the 5-year cancerspecific overall survival of patients with GPS 0 , low NLR, PLR 0 , PNI 1, low SII and high ALI were 83.19\%, 76.75\%, 80.92\%, $79.01 \%, 83.61 \%$ and $84.20 \%$, respectively. On the other hand, those of patients with GPS 1-2, high NLR, PLR 1-2, PNI 0, high SII and low ALI were 64.53\%, 40.18\%, 62.89\%, 67.40\%, $860.39 \%$ and $57.61 \%$, respectively. All of GPS $(\mathrm{P}<0.001)$, NLR $(\mathrm{P}<0.001)$, PLR $(\mathrm{P}<0.001)$, PNI $(\mathrm{P}=0.014)$, SII $(\mathrm{P}$ $<0.001)$ and ALI $(\mathrm{P}<0.001)$ were associated with a cancerspecific overall survival.

Comparison of the AUCs for cancer-specific overall survival prediction between these inflammation-based prognostic scores was shown in Figure 2. The AUCs of GPS, NLR, PLR, PNI, SII and ALI were 0.642, 0.643, 0.590, 0.608, 0.623 and 0.681 , respectively. ALI consistently had a higher AUC value in comparison with other inflammation-based scores.

The univariate analysis showed that gender, smoking status, histology, $\mathrm{pT}$ status, $\mathrm{pN}$ status, serum CEA level, serum CYFRA21-1 level, serum CRP level, GPS, NLR, PLR, PNI, SII and ALI were significant association with the 5-year cancer-specific survival (Table 3). All 13 clinicopathological characteristics and inflammation-based scores were further investigated in multivariate analysis. As shown in Table 3, gender ( $\mathrm{P}$ $=0.003)$, histology $(\mathrm{P}=0.001), \mathrm{pN}$ status $(\mathrm{P}=0.001)$, serum CEA level $(P=0.001)$, GPS $(P=0.009)$ and ALI $(P=0.026)$ were independent factors in predicting overall postoperative cancer specific survival, while others were not.

\section{Discussion}

The univariate analysis in this study demonstrated that the GPS, NLR, PLR, PNI, SII and ALI were significantly associated with overall cancer-specific survival of NSCLC patients who underwent potentially curative resection. However, the multivariate analysis showed that GPS and ALI were independently associated with overall cancer-specific survival. Moreover, the AUC analysis has shown that ALI was superior to other inflammation-based prognostic scores in terms of predictive accuracy in patients undergoing potentially curative resection for NSCLC. To our knowledge, this was the first report investigating the comparison of inflammation-based scores in operable patients with NSCLC, and showing GPS and ALI to be superior to other inflammation-based prognostic scores for the prediction of prognosis in patients with operable NSCLC.

Inflammation in the tumor microenvironment contributes substantially to tumor proliferation, angiogenesis, invasion, metastasis and survival, suggesting that the systemic inflammatory factors may act as the prognostic markers in NSCLC [1]. CRP is a non-specific inflammatory acute-phase protein, 
Table 2. The Relationships Between Clinical Characteristics and Each Inflammation-Based Score

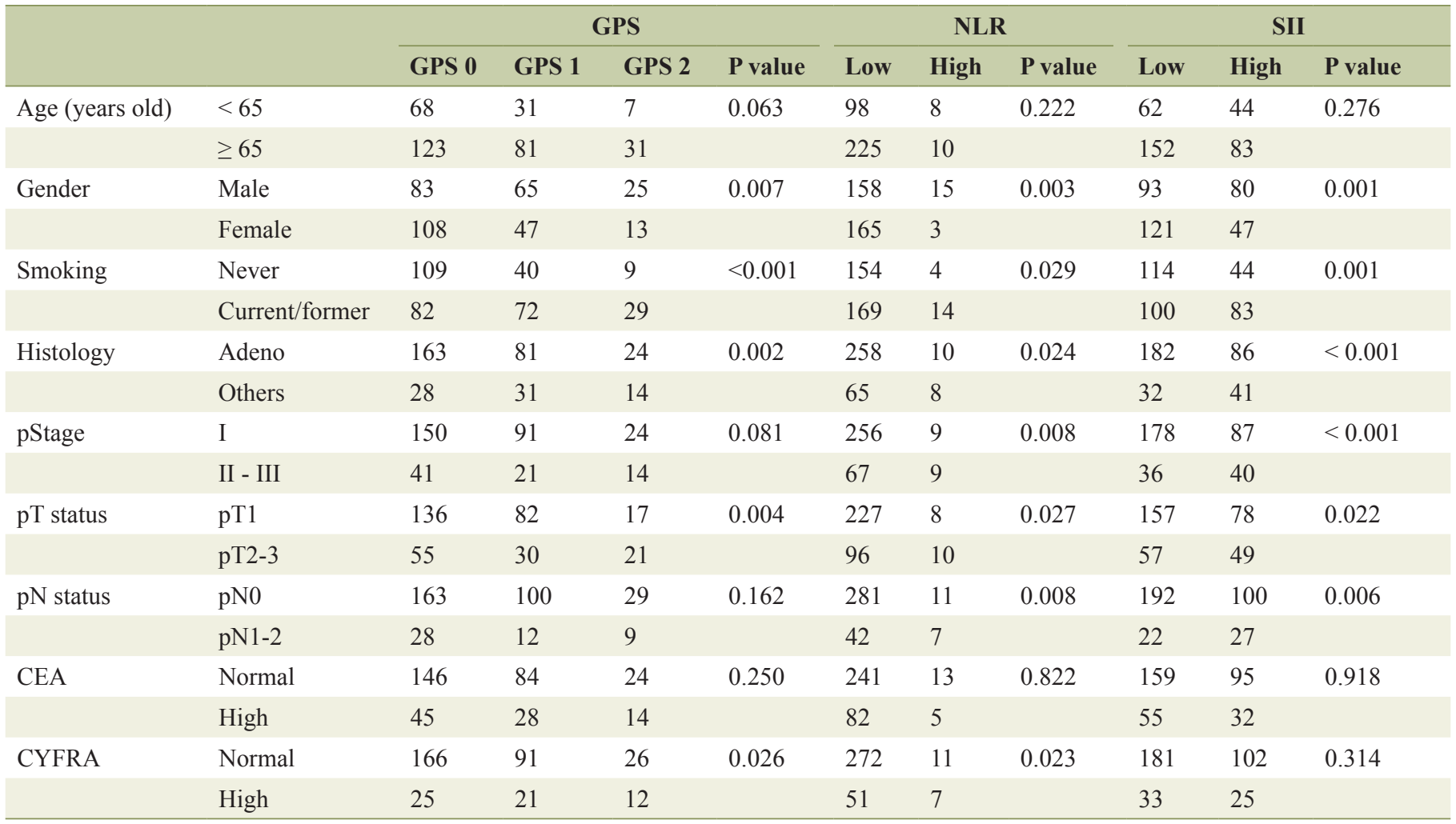

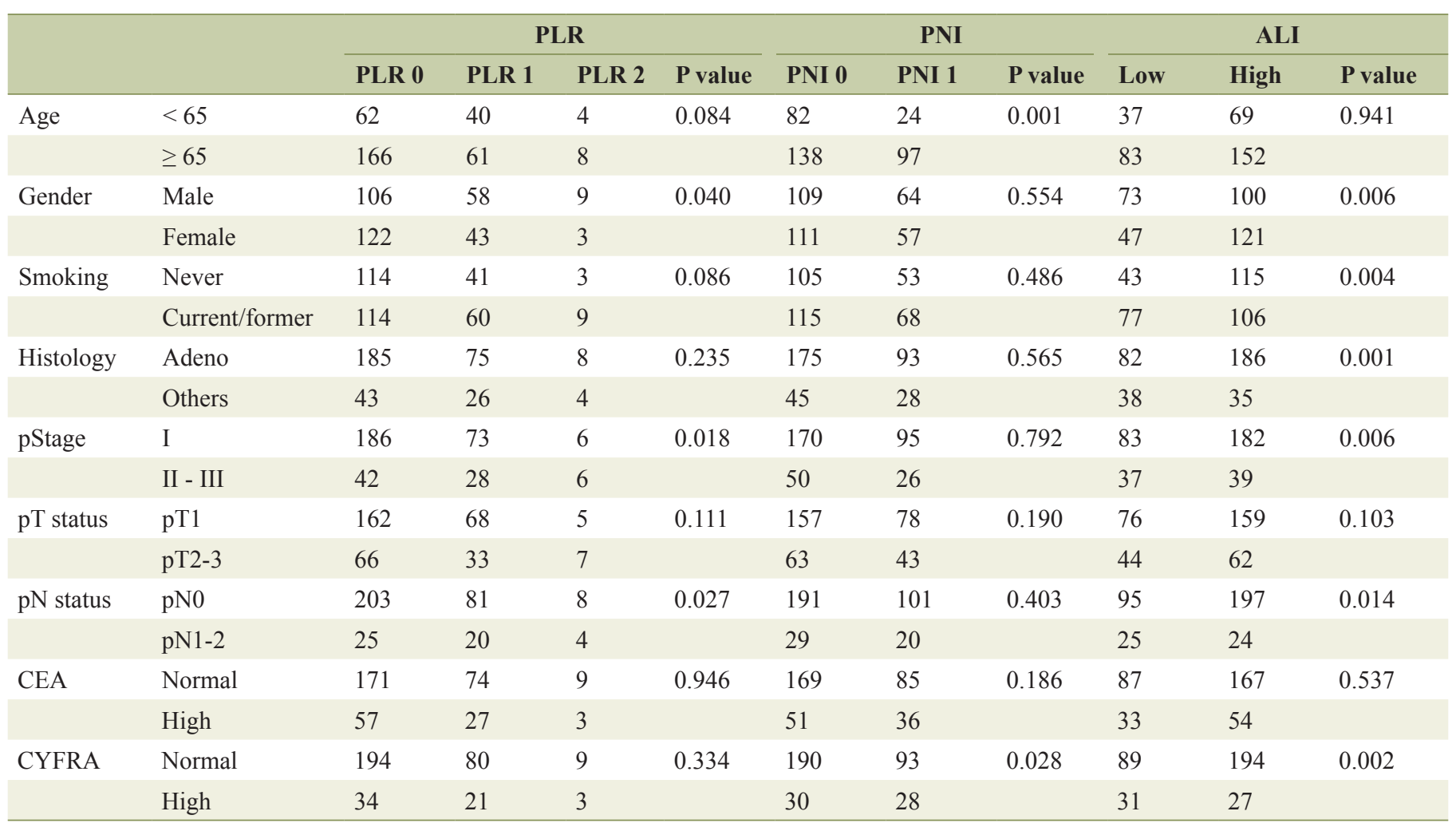

GPS: Glasgow prognostic score; NLR: neutrophil-to-lymphocyte ratio; PLR: platelet-to-lymphocyte ratio; PNI: prognostic nutritional index; SII: systemic immune-inflammation index; ALI: advance lung cancer inflammation index; CEA: carcinoembryonic antigen; CYFRA21-1: cytokeratin 19 fragments. 

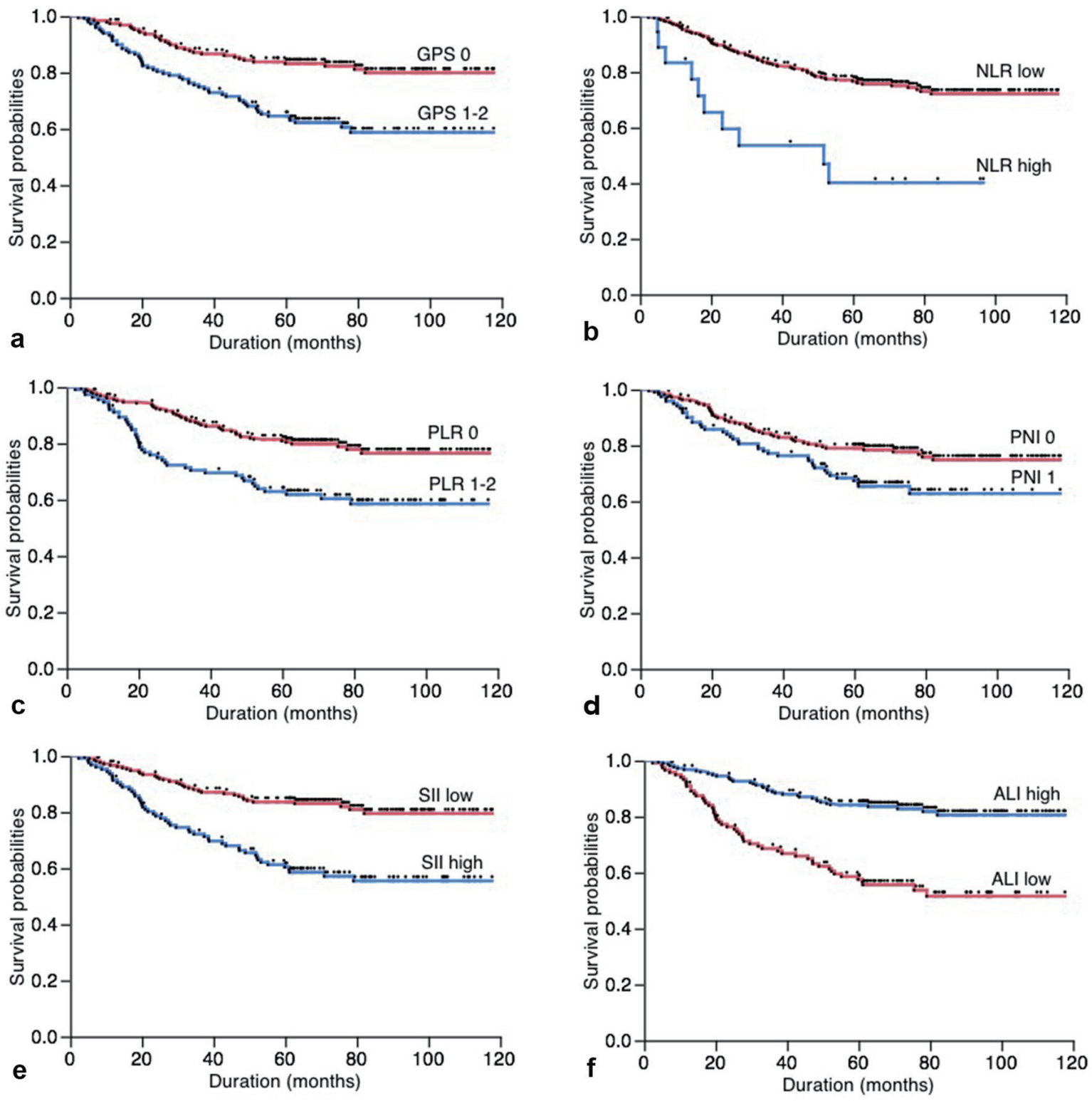

Figure 1. (a) Overall postoperative cancer-specific survival of patients based on Glasgow prognostic score (GPS, $P<0.001$ ); (b) neutrophil-to-lymphocyte ratio (NLR, $\mathrm{P}<0.001$ ); (c) platelet-to-lymphocyte ratio (PLR, $\mathrm{P}<0.001$ ); (d) prognostic nutritional index (PNI, $\mathrm{P}=0.014)$; (e) systemic immune-inflammation index (SII, $\mathrm{P}<0.001)$; (f) advance lung cancer inflammation index $(A L I, P<0.001)$.

and an elevated serum CRP level has been reported as an indicator of unfavorable prognosis in patients with NSCLC [13]. Among inflammation-based scores examined in this study, GPS was only based on CRP. Therefore, the superiority of GPS in multivariate analysis might be due to the prognostic power of CRP, at least in part. GPS was defined as the combination of CRP and albumin [2]. Nutritional status of cancer patients, commonly reflected by serum albumin, is also a determinant of survival in NSCLC [14]. Among inflammation-based scores examined in this study, in addition to GPS, PNI and ALI were defined to be based on albumin and other factors. Therefore, the prognostic power of albumin might be offset in multivari- ate analysis. Another factor to evaluate the nutritional status was body mass index (BMI). A meta-analysis showed that BMI had an inverse association with risk of cancer-specific survival in NSCLC [15]. Among inflammation-based scores examined, only ALI was based on BMI. Therefore, there is a possibility that the superiority of ALI in multivariate analysis and the AUC of ROC curve is also due to the prognostic power of BMI in part.

NLR has been reported to be a useful biomarker to measure the inflammatory status of immune system [3]. The neutrophil is known to be able to aid the proliferation and survival of malignant cells, promote angiogenesis and metastasis [3]. 


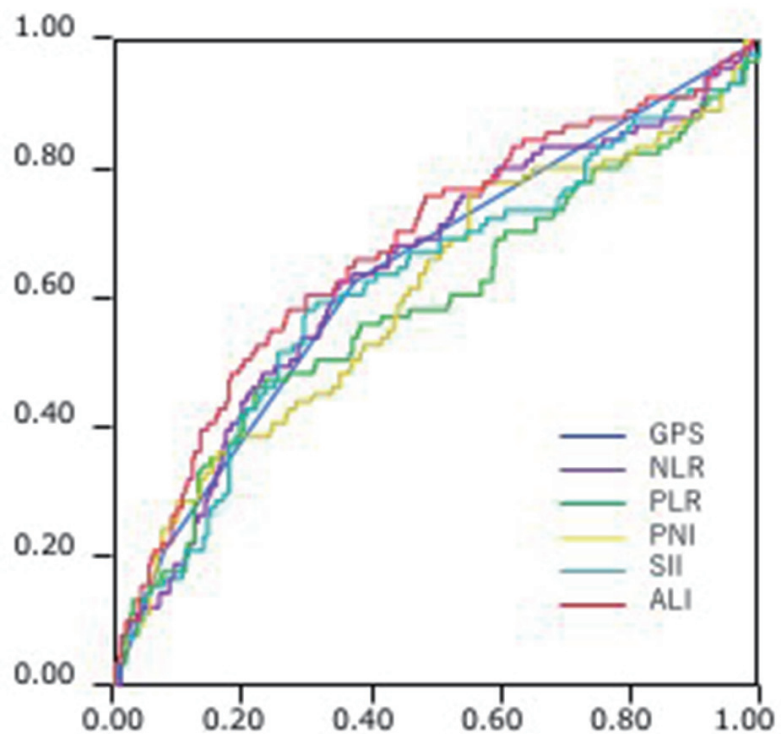

Figure 2. Comparison of the area under the receiver operating characteristics $(\mathrm{ROC})$ for outcome prediction between the inflammation-based prognostic scores.

Conversely, lymphocytes suppress tumor growth and invasion through their cytolytic activity [3]. Taken together, patients with high NLR have a relative lymphocytopaenia, and, as a result, may exhibit a poorer immune response to malignancy, thereby worsening their prognosis [3]. Among inflammationbased scores examined, in addition to NLR, SII and ALI were also based on NLR. Furthermore, PLR and PNI were defined as the combination of lymphocyte count and other factors. Therefore, there is a possibility that the prognostic power of NLR was offset in multivariate analysis.

Several limitations of this study should be acknowledged. First, the study involved a small sample size and single-center design. Second, this study's retrospective design renders it susceptible to selection and analytical biases. Nevertheless, our data indicated that preoperative GPS and ALI might represent independent prognostic factors in patients with resectable NSCLC. GPS and ALI are simple, inexpensive and they do not require extra equipment. Therefore, GPS and ALI should be taken into consideration in the therapeutic program for resectable NSCLC.

In conclusion, to the best of our knowledge, this is the first study to demonstrate that GPS and ALI appear to be superior to other inflammation-based prognostic scores in patients undergoing potentially curative resection for NSCLC.

\section{Conflict of Interest}

The authors have declared that no conflict of interest exists.

\section{References}

1. Peebles KA, Lee JM, Mao JT, Hazra S, Reckamp KL, Krysan K, Dohadwala M, et al. Inflammation and lung carcinogenesis: applying findings in prevention and treatment. Expert Rev Anticancer Ther. 2007;7(10):1405-

Table 3. Univariate and Multivariate Analyses

\begin{tabular}{|c|c|c|c|c|c|c|c|c|}
\hline & \multicolumn{4}{|c|}{ Univariate } & \multicolumn{4}{|c|}{ Multivariate } \\
\hline & \multirow{2}{*}{ Hazard ratio } & \multirow{2}{*}{ P value } & \multicolumn{2}{|c|}{$95 \%$ CI } & \multirow{2}{*}{ Hazard ratio } & \multirow{2}{*}{ P value } & \multicolumn{2}{|c|}{$95 \%$ CI } \\
\hline & & & Lower & Upper & & & Lower & Upper \\
\hline Gender & 0.329 & $<0.001$ & 0.204 & 0.514 & 0.367 & 0.003 & 0.183 & 0.717 \\
\hline Smoking status & 0.349 & $<0.001$ & 0.215 & 0.549 & 0.696 & 0.323 & 0.344 & 1.435 \\
\hline $\mathrm{pN}$ status & 0.330 & $<0.001$ & 0.211 & 0.534 & 0.409 & 0.002 & 0.242 & 0.704 \\
\hline CEA & 0.382 & $<0.001$ & 0.252 & 0.586 & 0.446 & 0.001 & 0.287 & 0.702 \\
\hline CYFRA21-1 & 0.353 & $<0.001$ & 0.229 & 0.556 & 0.835 & 0.502 & 0.501 & 1.428 \\
\hline GPS & 0.409 & $<0.001$ & 0.265 & 0.622 & 0.499 & 0.009 & 0.296 & 0.838 \\
\hline SII & 0.368 & $<0.001$ & 0.241 & 0.556 & 0.927 & 0.841 & 0.444 & 1.920 \\
\hline ALI & 0.312 & $<0.001$ & 0.204 & 0.473 & 0.444 & 0.026 & 0.217 & 0.907 \\
\hline
\end{tabular}

GPS: Glasgow prognostic score; NLR: neutrophil-to-lymphocyte ratio; PLR: platelet-to-lymphocyte ratio; PNI: prognostic nutritional index; SII: systemic immune-inflammation index; ALI: advance lung cancer inflammation index; CEA: carcinoembryonic antigen; CYFRA21-1: cytokeratin 19 fragments; Cl: confidence interval. 
1421.

2. Jin J, Hu K, Zhou Y, Li W. Clinical utility of the modified Glasgow prognostic score in lung cancer: A meta-analysis. PLoS One. 2017;12(9):e0184412.

3. Gu XB, Tian T, Tian XJ, Zhang XJ. Prognostic significance of neutrophil-to-lymphocyte ratio in non-small cell lung cancer: a meta-analysis. Sci Rep. 2015;5:12493.

4. Zhang H, Gao L, Zhang B, Zhang L, Wang C. Prognostic value of platelet to lymphocyte ratio in non-small cell lung cancer: a systematic review and meta-analysis. Sci Rep. 2016;6:22618.

5. Okada S, Shimada J, Kato D, Tsunezuka H, Teramukai S, Inoue M. Clinical significance of prognostic nutritional index after surgical treatment in lung cancer. Ann Thorac Surg. 2017;104(1):296-302.

6. Zhong JH, Huang DH, Chen ZY. Prognostic role of systemic immune-inflammation index in solid tumors: a systematic review and meta-analysis. Oncotarget. 2017;8(43):75381-75388.

7. Jafri SH, Shi R, Mills G. Advance lung cancer inflammation index (ALI) at diagnosis is a prognostic marker in patients with metastatic non-small cell lung cancer (NSCLC): a retrospective review. BMC Cancer. 2013;13:158.

8. Jiang K, Lei J, Li C, Shu K, Li W, Zhang Y, Li Z, et al. Comparison of the prognostic values of selected inflammation based scores in patients with medullary thyroid carcinoma: A pilot study. J Surg Oncol. 2017;116(3):281287.

9. Lei J, Li C, Shu K, Li W, Zhang Y, Li Z, Gong R, et al. Comparison of the prognostic values of preoperative in- flammation-based parameters in patients with breast cancer. J Surg Oncol. 2017;12(5):e0177137.

10. Pan QX, Su ZJ, Zhang JH, Wang CR, Ke SY. A comparison of the prognostic value of preoperative inflammationbased scores and TNM stage in patients with gastric cancer. Onco Targets Ther. 2015;8:1375-1385.

11. Yamamura K, Sugimoto H, Kanda M, Yamada S, Nomoto S, Nakayama G, Fujii T, et al. Comparison of inflammation-based prognostic scores as predictors of tumor recurrence in patients with hepatocellular carcinoma after curative resection. J Hepatobiliary Pancreat Sci. 2014;21(9):682-688.

12. Budczies J, Klauschen F, Sinn BV, Gyorffy B, Schmitt WD, Darb-Esfahani S, Denkert C. Cutoff Finder: a comprehensive and straightforward Web application enabling rapid biomarker cutoff optimization. PLoS One. 2012;7(12):e51862.

13. Jing X, Huang C, Zhou H, Li C, Fan L, Chen J, Zhang G, et al. Association between serum $\mathrm{C}$-reactive protein value and prognosis of patients with non-small cell lung cancer: a meta-analysis. Int J Clin Exp Med. 2015;8(7):1063310639.

14. Espinosa E, Feliu J, Zamora P, Gonzalez Baron M, Sanchez JJ, Ordon ez A, Espinosa J. Serum albumin and other prognostic factors related to response and survival in patients with advanced non-small cell lung cancer. Lung Cancer. 1995;12(1-2):67-76.

15. Wang J, Xu H, Zhou S, Wang D, Zhu L, Hou J, Tang J, et al. Body mass index and mortality in lung cancer patients: a systematic review and meta-analysis. Eur J Clin Nutr. 2018;72(1):4-17. 